\title{
ANALYSIS OF RESIDUAL STRESS STATES USING DIFFRACTION METHODS
}

\author{
W. REIMERS
}

Hahn-Meitner-Institut Berlin, Bereich Strukturforschung, 14109 Berlin, Germany

The possibilities and limitations of residual stress analysis using diffraction methods are discussed. Examples for the analysis of stress gradients in the near surface zone are given. The use of neutron diffraction and of high energy synchrotron radiation for the analysis of bulk stresses is demonstrated. Future trends of experimental residual stress analysis are outlined.

PACS numbers: $81.70 .-\mathrm{q}$

\section{Introduction}

The technological relevance of residual stress is mainly due to the superposition of internal and external stresses. Thus, the mechanical-technological properties of materials and technological components may be changed positively or negatively depending on the sign and value of the internal stresses. Since in many cases the residual stress distribution in materials and components can be varied by the choice of the processing parameters, it is often possible to optimize the stress distribution in view of the load stresses under in-service conditions and hence in view of the lifetime of the component.

Of course such an approach requires the knowledge of the residual stresses present in a component. For the experimental determination of residual stresses, the diffraction methods offer unique advantages. They are nondestructive and can be applied to all crystalline materials. Since the stress determination using diffraction methods is based on Bragg's equation, the internal strains can be determined on an absolute scale measuring the shifts either of the diffraction angle (angle-dispersive analysis) or of the energy of the diffracted radiation (energy-dispersive analysis). Moreover, diffraction methods are phase-specific, which is necessary for the determination and interpretation of stress states in composite materials. A further advantage of diffraction methods is the possibility of varying the information depth of the measurement systematically, e.g. by the choice of the radiation used. This point is of importance, because the residual stress distribution in materials and components is spatially inhomogeneous due to the mechanical equilibrium conditions. This fact, as well as the variety of technological processing techniques and the variety of applications of components lead to 
the demand for stress determinations ranging from the very near surface zone of components ( $\mu \mathrm{m}$ depth) down to the bulk material ( $\mathrm{cm}$ depth). The possibilities and the limitations of residual stress analysis in different information depths using diffraction methods are outlined within this paper.

\section{Principles of measuring techniques}

All residual stress analyses using diffraction methods are based on the measurement of interplanar lattice spacings $d^{h k l}$ of the lattice plane with Miller's indices $h k l$ using Bragg's equation

$$
n \lambda=2 d^{h k l} \sin \theta
$$

where $\lambda$ - wavelength, $\theta$ - diffraction angle. Due to the tensorial character of strains and stresses, the measurements of the interplanar lattice spacings have to be performed in different sample directions defined by $\varphi$ (azimuthal angle) and $\psi$ (inclination angle). In case of angle-dispersive measuring techniques $(\lambda=\mathrm{const}$ ), the strain values $\varepsilon_{\varphi \psi}^{h k l}$ are obtained from the shifts of the diffraction angle $\theta$.

In case of an energy-dispersive analysis of residual stresses, the Bragg equation has to be written as

$$
d^{h k l}=\frac{h c}{2 E^{h k l} \sin \theta}=\text { const } \frac{1}{E^{h k l}},
$$

where $h$ - Planck's constant, $c$ - light velocity, $E^{h k l}$ - energy of the reflection $h k l$. The strains can be determined from the lattice spacing respectively the energy by

$$
\varepsilon_{\varphi \psi}^{h k l}=\frac{d_{\varphi \psi}^{h k l}}{d_{0}^{h k l}}-1=\frac{E_{0}^{h k l}}{E_{\varphi \psi}^{h k l}}-1,
$$

where $E_{0}^{h k l}$ - energy of the reflection $h k l$ for the stress-free reference material. If the stress state in the analyzed sample volume is homogeneous and biaxial, which can often be assumed for the near surface zone, then the $\sin ^{2} \psi$-method [1] can be applied to calculate the stresses from the experimentally determined strains (Fig. 1).

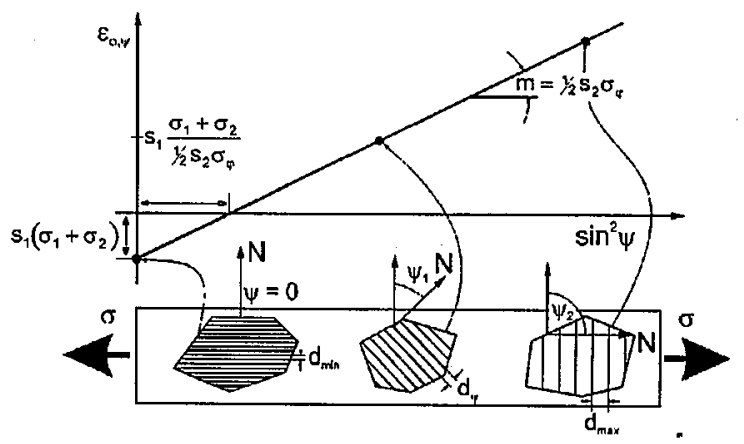

Fig. 1. $\sin ^{2} \psi$-method. 
According to the $\sin ^{2} \psi$-method, the stress value $\sigma_{\varphi}$ in the measuring direction defined by $\varphi$ is given by

$$
\sigma_{\varphi}=\frac{m}{\frac{1}{2} s_{2}^{h k l}},
$$

where $m$ - inclination of the graph (cf. Fig. 1), $\frac{1}{2} s_{2}^{h k l}$ - diffraction elastic constant. If triaxial stress states are present, which is typical in the bulk of material, the strain/stress tensors are given by

$$
\begin{aligned}
\varepsilon_{\varphi \psi} & =\varepsilon_{11} \cos ^{2} \varphi \sin ^{2} \psi+\varepsilon_{12} \sin 2 \varphi \sin ^{2} \psi+\varepsilon_{22} \sin ^{2} \varphi \sin ^{2} \psi+\varepsilon_{13} \cos \varphi \sin 2 \psi \\
& +\varepsilon_{33} \cos ^{2} \psi+\varepsilon_{23} \sin \varphi \sin 2 \psi \\
\sigma_{i j}= & \frac{E}{1+\nu}\left(\varepsilon_{i j}+\frac{\nu}{1-2 \nu} \delta_{i j} \sum_{i=1}^{3} \varepsilon_{i i}\right), \quad \delta_{i j}=0, \delta_{i i}=1(i, j=1,2,3) .
\end{aligned}
$$

Whereas the $\sin ^{2} \psi$-method is nearly insensitive to the value $d_{0}^{h k l}$ of the stress-free material, the determination of absolute stress values in case of triaxial stresses requires the precise knowledge of $d_{0}^{h k l}$. This value then has to be determined either by measurements on a stress-free reference material or from the application of mechanical boundary conditions.

\section{Analysis of very near surface stress gradients}

Near-surface stress gradients have to be expected in thin coatings, but also as a consequence of surface working processes, e.g. grinding. The presence of stress gradients in the normal to the surface direction manifests itself in a curvature of the $d_{\varphi \psi}^{h k l}$ versus $\sin ^{2} \psi$ graph. This is due to the fact that the penetration depth of the radiation varies with the inclination angle $\psi$ as a consequence of the absorption of the radiation. For the evaluation of such nonlinear $\varepsilon_{\varphi \psi}^{h k l}$ strain distributions, different methods have been proposed (e.g. [2-4]). A promising new procedure for analyzing stress gradients in the near surface zone using X-rays or synchrotron radiation is based on the systematic variation of the penetration depth at defined $\varphi, \psi$-measuring directions. Therefore, the information depth $\tau$ is varied rotating the sample by the angle $\eta$ around the measuring direction $g_{\varphi \psi}$ (scattering vector) (Fig. 2)

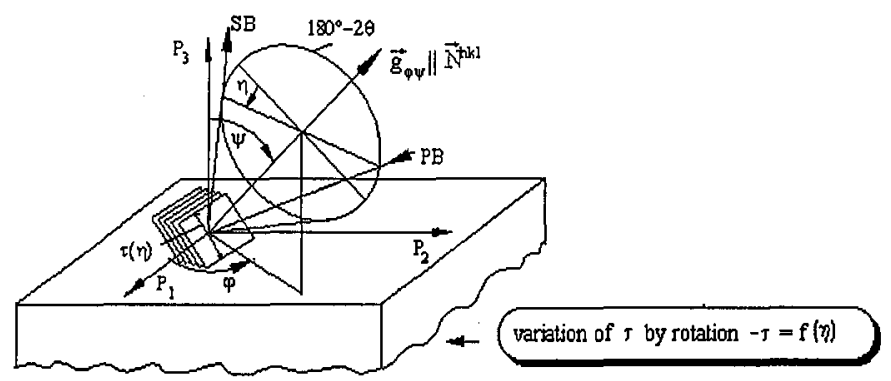

Fig. 2. Geometry of the $\eta$-measuring technique. 

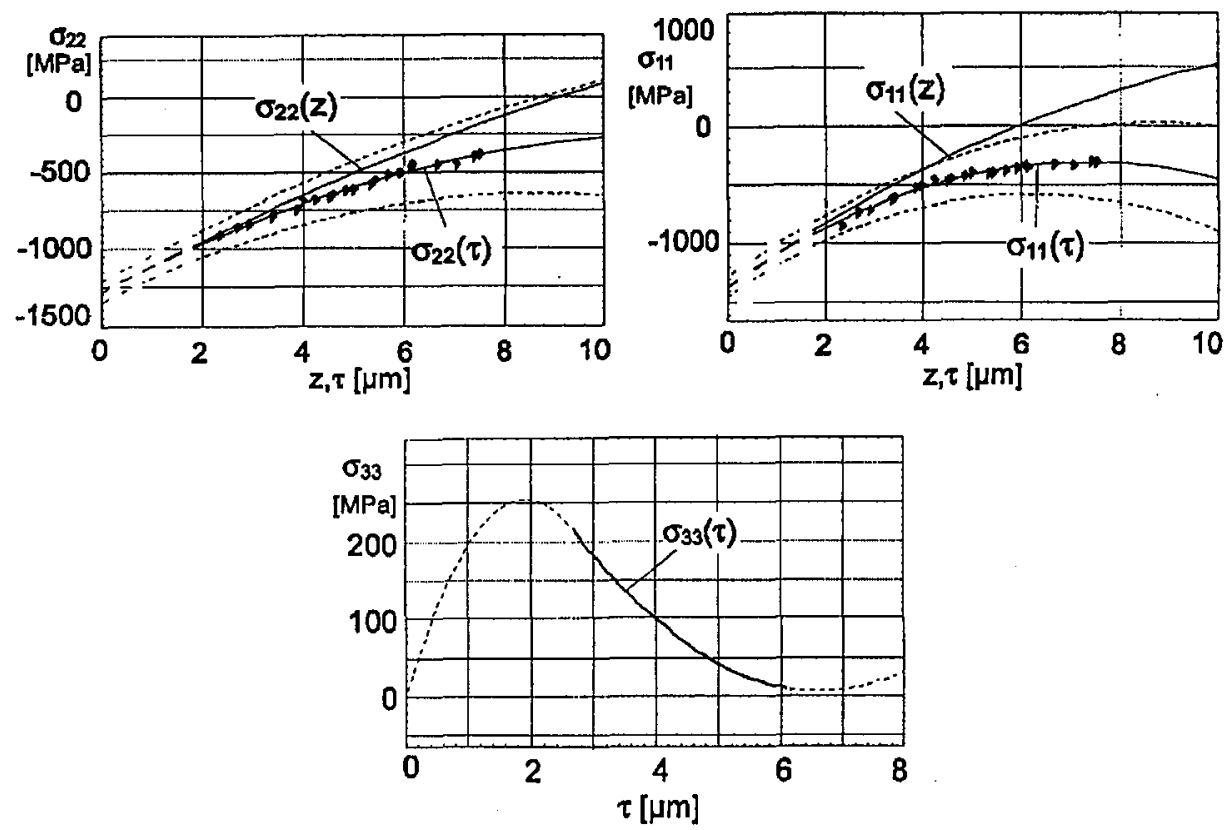

Fig. 3. Near surface residual stress fields. The dashed part in the front range of the $\sigma_{33}(\tau)$ curve was completed by the demand $\sigma_{33}(z, \tau=0)=0$.

$$
\tau_{\eta}=\frac{\sin ^{2} \theta-\sin ^{2} \psi+\cos ^{2} \theta \sin ^{2} \psi \sin ^{2} \eta}{2 \mu \sin \theta \cos \psi}
$$

where $\mu$ is the linear attenuation coefficient. Measurements were performed on pendulum ground $\mathrm{Si}_{3} \mathrm{~N}_{4}$ ceramics. Figure 3 shows the results using the formalism described in Ref. [4]. The transverse stress component $\sigma_{22}(\tau)$ is found to be $100 \ldots 200 \mathrm{MPa}$ higher than those of the $\sigma_{11}(\tau)$ component in grinding direction as well of the normal component $\sigma_{33}(\tau)$.

\section{Analysis of near-surface stress gradients}

Whereas the information depth of conventional X-ray diffraction measurements using the characteristic wavelength of the typical anode materials $\mathrm{Co}, \mathrm{Cu}$ or $\mathrm{Cr}$ is limited to some ten $\mu \mathrm{m}$ in case of metallic materials, the information depth can be extended to approximately $200 \mu \mathrm{m}$ using the white X-radiation $[5,6]$. For analyzing the energy value corresponding to the reflecting lattice planes $h k l$ and their $d^{h k l}$-values an energy-dispersive detector is required. Figure 4 shows the spectrum of a steel sample. Figure 4 reveals that the energy-dispersive analysis allows the simultaneous measurement of several reflections, each registered at a different energy and therefore each representing a different information depth. Whereas for metallic materials penetration depths of approximately $100 \mu \mathrm{m}$ are possible, in case of ceramic materials several $\mathrm{mm}$ are accessible (Fig. 5). 


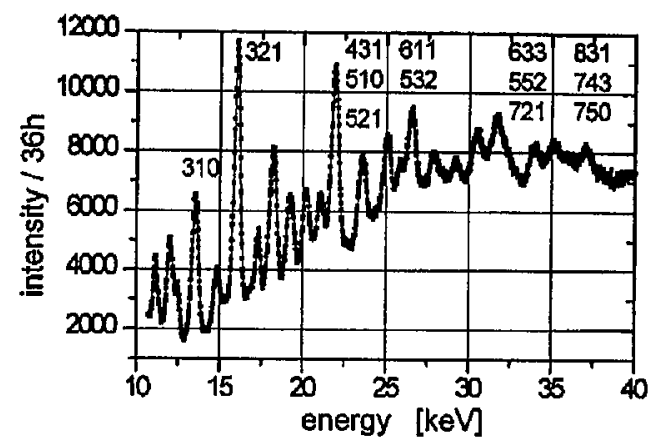

Fig. 4. Spectrum of a steel sample, registered at $2 \theta=60^{\circ}$.

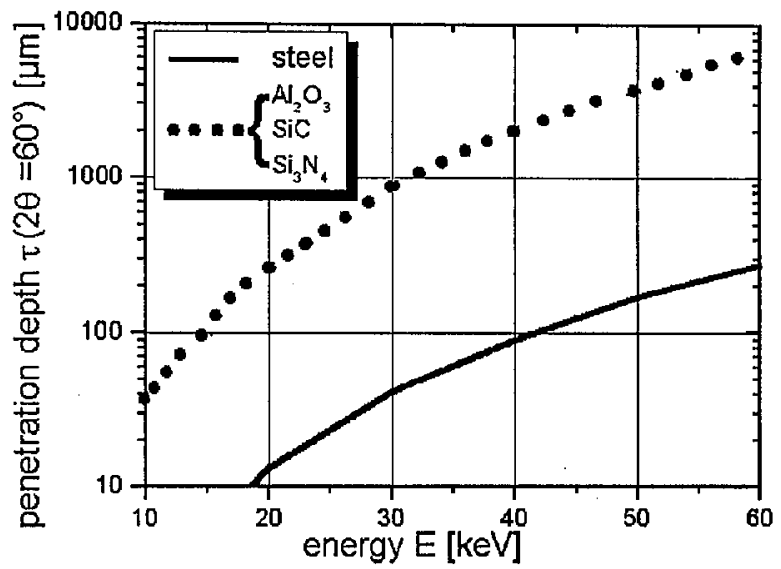

Fig. 5. Penetration depth for different materials.

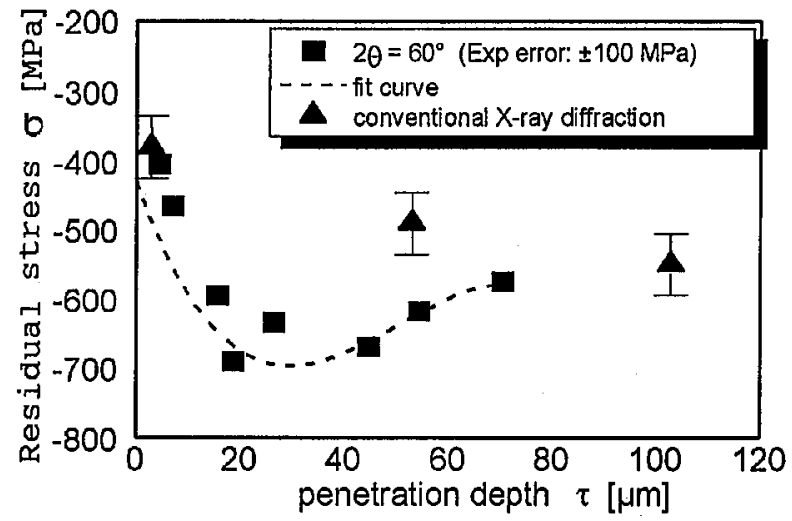

Fig. 6. Residual stresses in case hardened steel.

Stress depth profiles over a scale of several hundred $\mu \mathrm{m}$ are typical of surface treatments such as rolling, shot peening, and case hardening. Figure 6 shows the results of an energy dispersive analysis compared to conventional diffraction mea- 
surements, that were repeated after etching, for the case of a case hardened steel. The results are in good agreement. Further application examples for high-energy $\mathrm{X}$-ray diffraction residual stress analyses can be found in [6].

\section{Residual stress analysis in the bulk of components using neutron diffraction}

Due to the small attenuation coefficient of neutrons for most materials of technical importance a penetration depth in the range of $\mathrm{cm}$, in the case of $\mathrm{Al}$ even ten $\mathrm{cm}$, can be achieved. Since the neutrons are diffracted along their whole pass through the material, the gauge volume is defined by diaphragms for the incoming and the diffracted beam. Moreover, for the $d^{h k l}$-spacing measurement it is necessary to select a reflection $h k l$ which exhibits a reflecting angle $2 \theta \approx 90^{\circ}$ in order to ensure that the same volume is investigated during the measurement in all sample directions (Fig. 7). For intensity reasons the minimum gauge volume for neutron diffraction is approximately $1 \mathrm{~mm}^{3}$. Due to the comparatively high costs of reactor neutrons, the use of neutron diffraction for residual stress analysis is limited
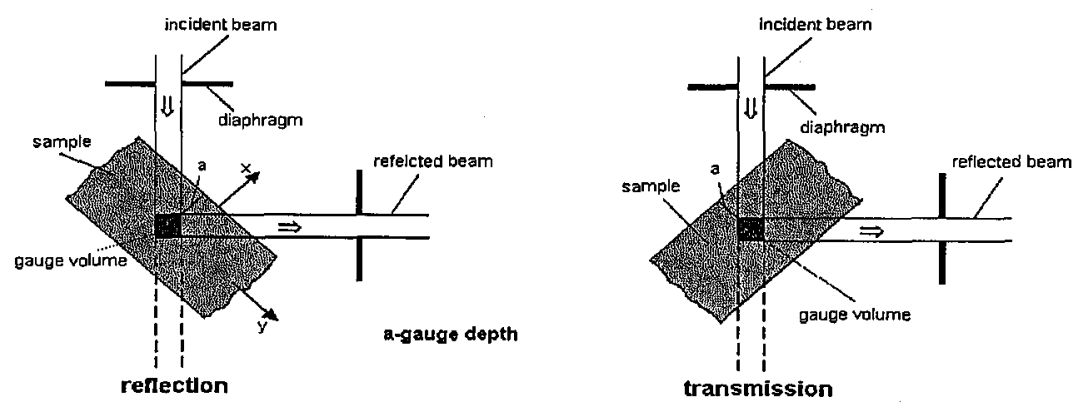

Fig. 7. $90^{\circ}$-scattering technique.
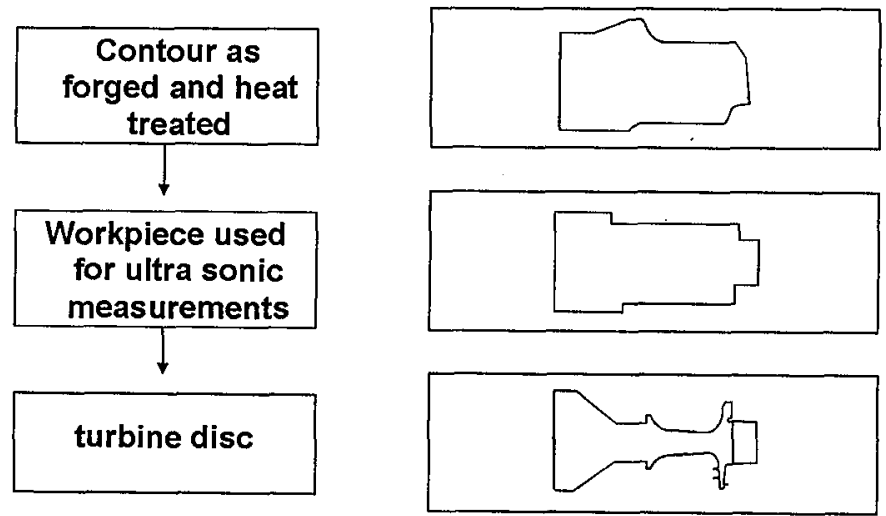

Fig. 8. Residual stress analysis in a Ni-base turbine disc. 

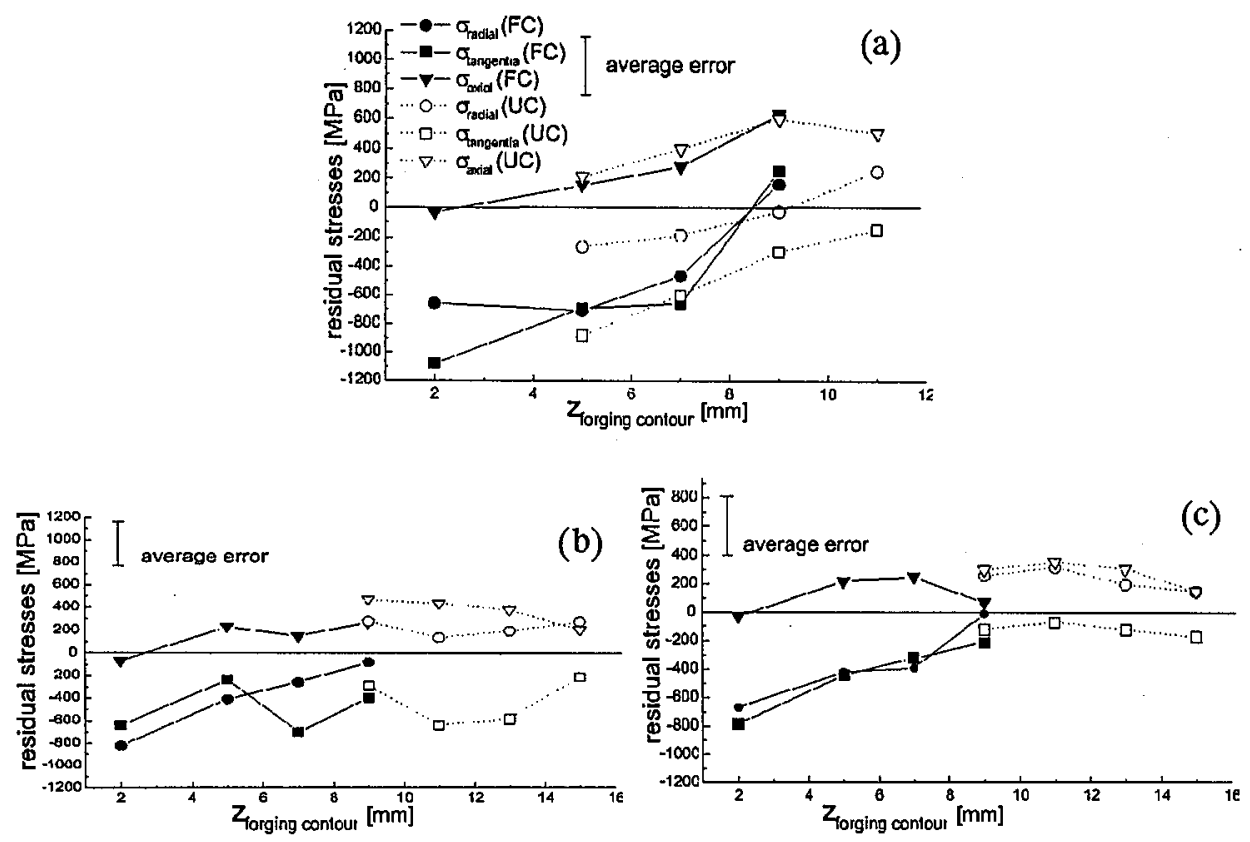

Fig. $9(a, b, c)$. Depth profiles of the residual stresses in aeroengine turbine discs.

to those cases where other methods fail. Typical examples for the necessity to use neutron diffraction are:

- Analysis of triaxial stress states in metal-ceramic composites, especially with strongly X-ray absorbing metallic partners such as tungsten [7].

- Validation of finite element calculations in technical components, e.g. crank shafts [8].

- Analyses of residual stress in large components [9].

Since neutron diffraction is a nondestructive technique, it is also possible to follow the residual stress development for subsequent manufacturing steps (Fig. 8). Figure 9 demonstrates that different measuring series fit well together.

\section{Residual stress analysis in bulk of components using high-energy $\mathrm{X}$-ray diffraction}

Penetration depths of $10 \mathrm{~mm}$ can also be achieved by high-energy X-rays of approximately $150 \mathrm{kV}$. The difference to the neutron diffraction case is given by the necessary low $2 \theta$-angle which is required because of the steep decrease in the atomic form factor for high-energy X-rays. This leads to two consequences: first of all, only sample geometries which allow the measurement in transmission geometry can be analyzed and secondly, the geometry of the gauge volume is no more a cube but a parallelepiped, which may lead to problems in cases where strongly inhomogeneous stress distributions are present. Moreover, due to the enormous intensity, especially 
at synchrotron sources of the third generation, e.g. the European Synchrotron Radiation Facility (ESRF), Grenoble, the diaphragm slits defining the width of the incoming and diffracted beam can be reduced to less than $100 \mu \mathrm{m}$, thus yielding a volume element of approximately $1.5 \times 0.08 \mathrm{~mm}^{2}$ at $2 \theta=10^{\circ}$ [10]. Such a gauge volume geometry is well suited to analyze the residual stresses in layer compounds, e.g. the stress distribution in a ceramic thermal barrier coating and in the interface layer of a coated turbine blade (Fig. 10).
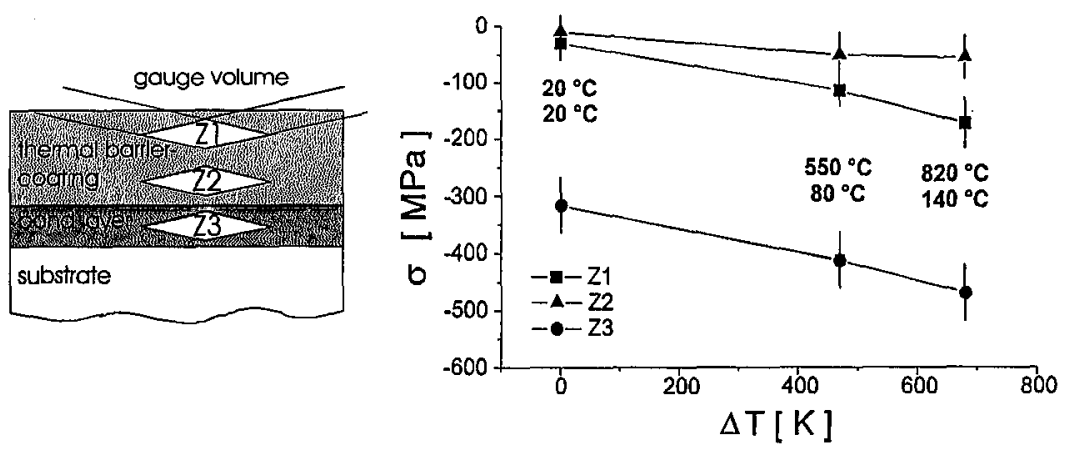

Fig. 10. Stress analysis with high-energy synchrotron radiation in a thermal barrier coating.

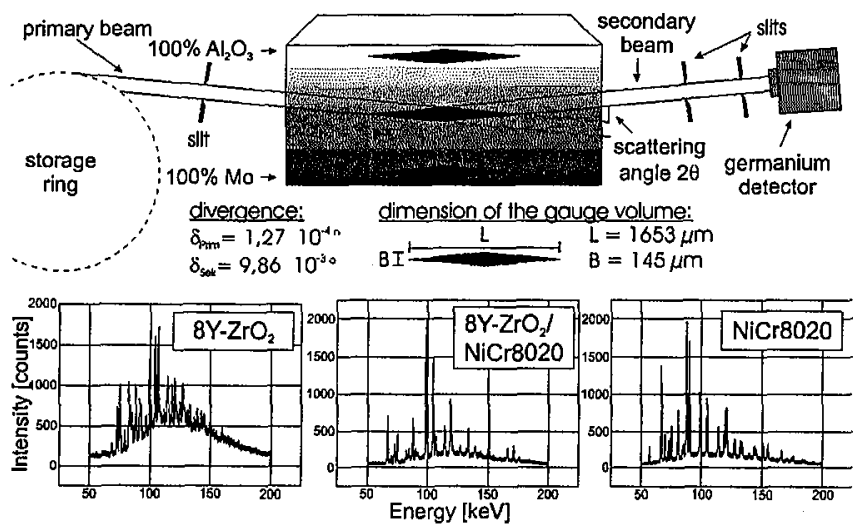

Fig. 11. Diffraction experiment with high-energy synchrotron radiation on metalceramic functionally graded materials.

Further residual stress studies using the high-energy photons delivered by the beam line ID 15 of the ESRF were performed on metal-ceramic functionally graded materials [11]. The experimental setup and typical diffraction spectra are shown in Fig. 11.

Figure 12 shows that the phase-specific stress of both partners in a metal-ceramic graded composite can be analyzed even over the thickness of one-composite interlayer of the sample. 


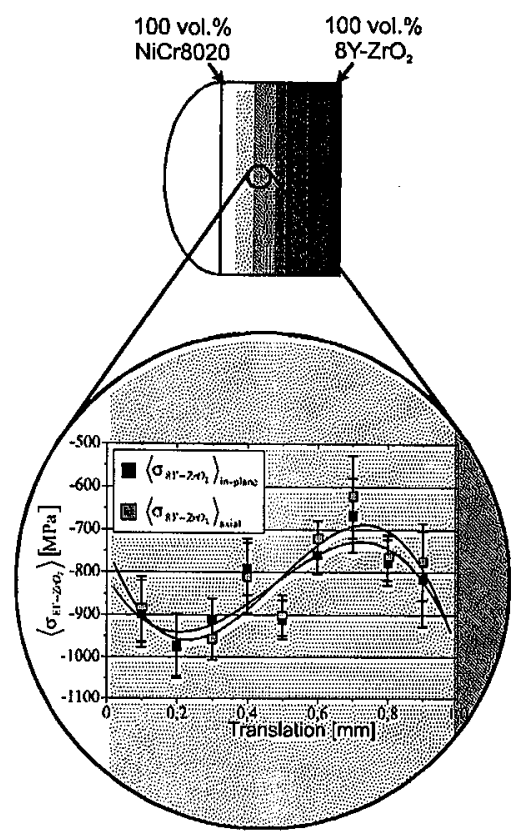

Fig. 12. Phase-specific residual stress distribution in one-composite interlayer.

\section{Outlook}

The experimental analysis of residual stresses is an expanding field due to the increasing demands in residual stress investigations, which arise from the increasing variety of materials, especially composite materials, but also from the rapid development of process technologies.

In parallel, the possibilities of residual stress analysis by diffraction methods are also increasing. There are new optical X-ray devices available, e.g. glass capillaries, multilayer collimators, which will lead to an enhanced local resolution of stress analyses, necessary e.g. in microelectronic devices of micromechanical components. The use of two-dimensional position-sensitive detectors for X-ray residual stress analysis will lead to new measuring strategies especially for the stress analysis in textured materials. The high intensity of photons delivered by synchrotron facilities will open new fields for the application of residual stress analysis. Of special importance is the field of in situ analyses of residual stress formation.

\section{References}

[1] E. Macherauch, P. Müller, Z. Angew. Phys. 13, 305 (1961).

[2] V. Hauk, Adv. X-ray Anal. 27, 101 (1984).

[3] B. Eigenmann, B. Scholtes, E. Macherauch, in: Proc. ICRS 2, Nancy 1998, Eds.

G. Beck, S. Denis, A. Simon, Elsevier Applied Sci., London 1989, p. 27.

[4] C. Genzel, W. Reimers, Phys. Status Solidi A 166, 751 (1998).

[5] H. Ruppersberg, Adv. X-ray Anal. 37, 235 (1994). 
[6] G. Brusch, Ph.D. Thesis, HMI-B 550, 1998.

[7] A. Pyzalla, W. Reimers, Physica B 234-236, 974 (1997).

[8] C. Achmus, W. Reimers, H. Wohlfahrt, Mat. Prüf. 40, 88 (1998).

[9] A. Pyzalla, W. Reimers, in: Mat-tec 97, Reims 1997, Ed. A. Lodini, Gournay-sur-Marne 1997, p. 171.

[10] W. Reimers, M. Broda, G. Brusch, D. Dantz, K.-D. Liss, A. Pyzalla, T. Schmackers, T. Tschentscher, J. Nondestr. Eval. 17, 129 (1998).

[11] D. Dantz, C. Genzel, W. Reimers, in: Proc. 5th Int. Symp. on Functionally Graded Materials, Dresden 1998, in print. 